University of Wollongong

Research Online

Faculty of Engineering and Information

Faculty of Engineering and Information

Sciences - Papers: Part A

Sciences

$1-1-2013$

\title{
Analytical nonlinear reluctance model of a single-phase saturated core fault current limiter
}

Philip A. Commins

University of Wollongong, pcommins@uow.edu.au

Jeffrey W. Moscrop

University of Wollongong, jeffm@uow.edu.au

Follow this and additional works at: https://ro.uow.edu.au/eispapers

Part of the Engineering Commons, and the Science and Technology Studies Commons

Research Online is the open access institutional repository for the University of Wollongong. For further information contact the UOW Library: research-pubs@uow.edu.au 


\title{
Analytical nonlinear reluctance model of a single-phase saturated core fault current limiter
}

\author{
Abstract \\ A saturated core fault current limiter $(F C L)$ is a device that is designed to limit the fault currents in \\ electrical energy networks and consequently, protect existing network equipment from damage. Due to \\ complex nonlinear magnetic properties, the performance of saturated core FCLs has largely been \\ characterized through experimentation and finite-element analysis simulations. Although both of these \\ techniques are quite accurate, they are time consuming and do not describe the behavior of FCLs in \\ actual electrical networks. This has led to an increasing demand for an accurate analytical model that is \\ suitable for transient network analyses. This paper presents the development of an analytical model of a \\ single-phase open-core FCL, which accurately describes the nonlinear magnetic properties of the FCL \\ through a reduced reluctance approach. The extension of this model to other saturated core FCL \\ arrangements (such as closed core) is also discussed. (C) 1986-2012 IEEE.
}

\section{Keywords}

saturated, nonlinear, core, analytical, fault, current, limiter, phase, single, model, reluctance

Disciplines

Engineering | Science and Technology Studies

\section{Publication Details}

Commins, P. A. \& Moscrop, J. W. (2013). Analytical nonlinear reluctance model of a single-phase saturated core fault current limiter. IEEE Transactions on Power Delivery, 28 (1), 450-457 


\title{
Analytical Nonlinear Reluctance Model of a Single Phase Saturated Core Fault Current Limiter
}

\author{
Philip A. Commins, Jeffrey W. Moscrop Member, IEEE
}

\begin{abstract}
A saturated core Fault Current Limiter (FCL) is a device that is designed to limit the fault currents in electrical energy networks and consequently, protect existing network equipment from damage. Due to complex nonlinear magnetic properties, the performance of saturated core FCLs has largely been characterised through experimentation and Finite Element Analysis (FEA) simulations. Although both of these techniques are quite accurate, they are time consuming and do not describe the behaviour of FCLs in actual electrical networks. This has led to increasing demand for an accurate analytical model that is suitable for transient network analyses. This paper presents the development of an analytical model of a single-phase open-core FCL, which accurately describes the nonlinear magnetic properties of the FCL through a reduced reluctance approach. The extension of this model to other saturated core FCL arrangements (such as closed-core) is also discussed.
\end{abstract}

Index Terms-Analytical models, fault current limiters, magnetic circuits, magnetic flux, nonlinear magnetics, power system protection

\section{INTRODUCTION}

$\mathbf{T}$ HE increasing demands placed on modern power systems [1] have not only led to increased occurrences of fault currents in networks, but also to increases in both fault current levels and equipment sensitivity to fault currents. Although new technologies and improvements in infrastructure continue to emerge, Fault Current Limiting (FCL) devices are becoming an increasingly necessary technology to protect existing infrastructure and improve network availability for consumers. There are several different FCL technologies that are currently attracting worldwide attention from both researchers and commercial engineering companies. This paper focuses on one particular technology, the saturated core FCL.

The saturated core FCL utilises the change in permeability between saturated and unsaturated states of the core material to provide both a low steady-state insertion impedance (so as not to load the network during normal operation) and a high transient impedance during fault conditions (for current limiting). The major advantages of this technology are that it provides instantaneous reaction to a fault event and instantaneous recovery. A DC current carrying winding is used to initially drive the cores into saturation, with separate windings

The research presented in this paper was supported under the Australian Research Council's (ARC) Linkage Projects funding scheme (project number LP100200461). Grateful acknowledgment is given to the ARC, along with the project partner organisations Zenergy Power and Powerlink Queensland, for their support.

P. A. Commins is with the Faculty of Engineering, University of Wollongong, Wollongong, NSW 2522, AUSTRALIA (e-mail: pcommins@uow.edu.au).

J. W. Moscrop is with the Faculty of Engineering, University of Wollongong, Wollongong, NSW 2522, AUSTRALIA (e-mail: jeffm@uow.edu.au). used to carry the AC load current of the circuit to be protected. Under normal steady-state load conditions the AC current is not large enough to drive the cores out of saturation, hence the AC coils behave like standard air core reactors. However, during a fault event the high $\mathrm{AC}$ fault currents drive the cores out of saturation, resulting in a significant increase in impedance that effectively limits the current. In a singlephase device, two AC coils and cores are necessary to limit both the positive and negative half cycles of the current. A more thorough description of the principles of operation of a saturated core FCL is given in [2].

As FCL technology becomes more viable for electrical utilities, there is an increasing demand for accurate simulations that can demonstrate the effects an FCL has on other network equipment. Although there have already been several experimental and Finite Element Analysis (FEA) studies on the performance characteristics of saturated core FCLs [2][5], network simulation packages (such as PSCAD and other ElectroMagnetic Transient Programs) cannot easily be coupled to an electromagnetic FEA simulator. Hence, there is growing need for an accurate analytical model of the FCL, which can be easily incorporated into transient network simulation packages. The development of an accurate analytical model has already received some attention [6], [7]; however, the models developed to date do not include the AC to DC coupling effects of the device. It has been found that a "transformer" coupling effect exists between the AC and DC windings during a fault [5], which can have a significant impact on the performance of the FCL.

This paper presents the development of an analytical "reluctance" model of a saturated core FCL that describes the full nonlinear range of magnetic operation as well as the $\mathrm{AC}$ to DC coupling effects of the device. The model is based on the magnetic circuit concept [8], with the geometry of the FCL represented by an equivalent magnetic circuit that includes all significant flux paths. A simple single-phase air-core geometry is initially examined, with the concept then extended to an open-core arrangement (i.e. the AC coils enclose separate iron cores that do not have a return path). Further extension of the model to other arrangements, such as closed-core, is also discussed. The model is validated against FEA simulations and shown to provide an accurate representation of a saturated core FCL, which with further development can be integrated with transient network simulation packages.

\section{AIR-CORE EQUIVALENT MODEL}

The initial development of the analytical reluctance model was carried out on a simple single-phase air-core arrangement, 
as shown in Fig. 1. This arrangement consists of two AC current carrying windings (AC coils) that are placed side by side and encompassed by a single DC current carrying winding (DC coil). Although this arrangement is not useful as a saturated core FCL (i.e. the AC coils are equivalent to air-core reactors and the DC coil serves no practical purpose), it is beneficial for model development as the significant flux paths are identical to the iron-core cases yet the associated reluctances are constant. Hence, the magnetic circuit described in this section can easily be extended to include the nonlinear reluctances associated with other iron-core arrangements (as shown in Section III).

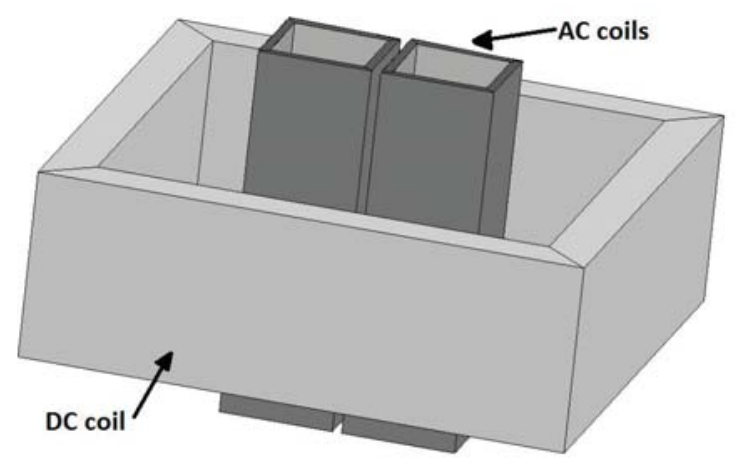

Fig. 1. Single-Phase Air-Core Arrangement

The significant flux paths (and associated reluctances) for the arrangement of Fig. 1 are identified in Fig. 2, where $\Re_{c}$ represents the reluctance of the flux paths inside the AC coils (i.e. the air-cores), $\Re_{i}$ represents the reluctance of the remaining flux paths inside the DC coil (identical on each side), $\Re_{o}$ represents the reluctance of the flux paths outside the DC coil (identical on each side), $\Re_{y}^{\prime}$ represents the reluctance of the flux paths between the two AC coils (top \& bottom), and $\Re_{a}^{\prime}$ represents the reluctance of the flux paths that link the inner AC loop with the other paths (top and bottom and identical on each side). The equivalent magnetic circuit for this arrangement is shown in Fig. 3, where $N I_{d c}, N I_{a c 1}$ and $N I_{a c 2}$ represent mmf due to the DC coil and two AC coils respectively. Note also that the $\Re_{y}^{\prime}$ and $\Re_{a}^{\prime}$ reluctances can be lumped in the equivalent circuit (as shown in Fig. 3).

One important feature of the magnetic circuit shown in Fig. 3 is that it is symmetrical, with $\Re_{a}, \Re_{i}, \Re_{o}$ and $N I_{d c}$ identical on the left and right sides of the circuit. In particular, $N I_{d c}$ on each side represents the same physical mmf. The reason that both sides are required is so that the flux due to each AC coil is accurately described - although the circuit elements are the same on each side, the flux in each corresponding path is only the same when $N I_{a c 1}=N I_{a c 2}$ (which is not the case during normal operation). Hence, the total flux linking the DC coil is the sum of the flux through $\Re_{O}$ on the left side and the flux through $\Re_{o}$ on the right side.

The values of the reluctances shown in Fig. 3 can be calculated using standard circuit analysis techniques. In general, all of the reluctances can be determined via flux measurements (using either FEA or experimentation) under three different

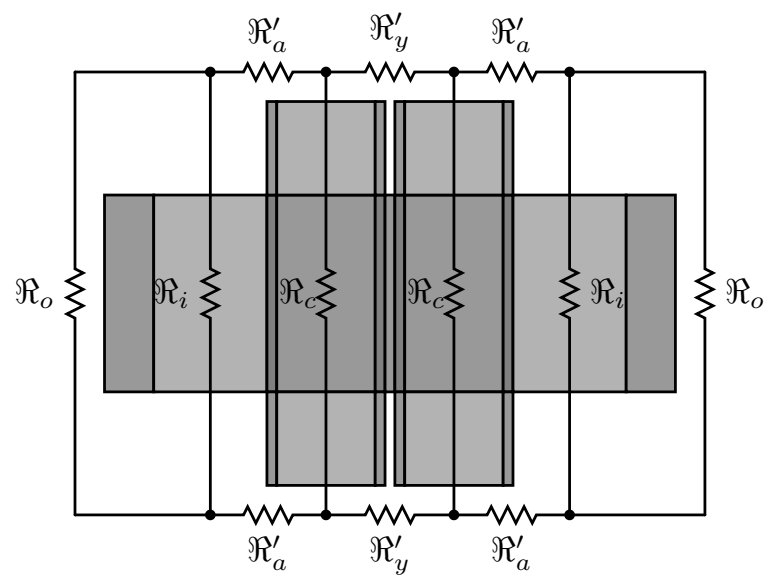

Fig. 2. Equivalent Reluctance Paths

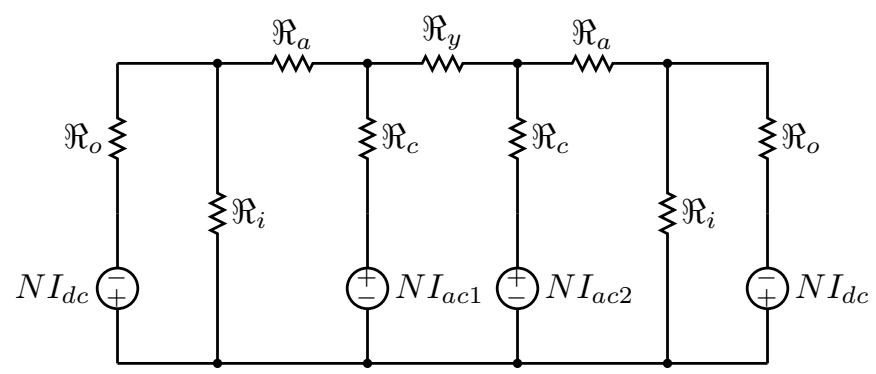

Fig. 3. Equivalent Magnetic Circuit

test conditions:

- Test 1: $N I_{d c} \neq 0$ and $N I_{a c 1}=N I_{a c 2}=0$;

- Test 2: $N I_{d c}=0$ and $N I_{a c 1}=N I_{a c 2} \neq 0$;

- Test 3: $N I_{d c}=0$ and $N I_{a c 1}=-N I_{a c 2} \neq 0$.

Test 1 and Test 2 are used to determine the values of $\Re_{i}$ and $\Re_{o}$, along with the series combination of $\Re_{c}+\Re_{a}\left(=\Re_{c a}\right)$. Test 3 is then used to determine the individual values of $\Re_{c}$ and $\Re_{a}$, along with $\Re_{y}$.

\section{A. Test 1}

The only source of mmf in this test is $N I_{d c}$, which results in a completely balanced circuit. Hence, the flux paths between the two AC coils are no longer significant and the equivalent circuit reduces to two separate sides that have identical reluctance and flux in each corresponding path (as shown in Fig. 4). The nominal direction of each flux path is as indicated in Fig. 4. The flux linkage of each coil is measured during the test.

Through simple circuit analysis:

$$
\begin{aligned}
\Re_{i}\left(\phi_{o 1}-\phi_{c 1}\right) & =N I_{d c}-\Re_{o} \phi_{o 1} \\
\text { and } \Re_{c a} & =\frac{\Re_{i}\left(\phi_{o 1}-\phi_{c 1}\right)}{\phi_{c 1}}
\end{aligned}
$$

Note also that $\phi_{o 1}$ is equal to one half of the measured flux linkage of the DC coil and $\phi_{c 1}$ is equal to the measured flux linkage of either AC coil. 


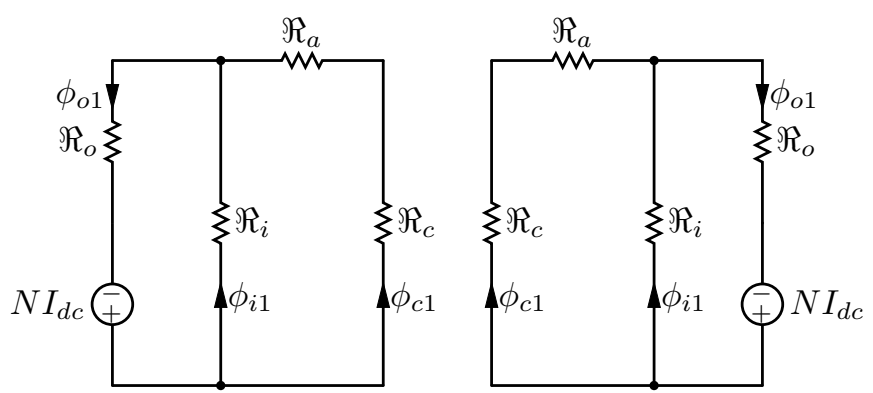

Fig. 4. Equivalent Circuit for Test 1

\section{B. Test 2}

In this test the sources of mmf are restricted to the $\mathrm{AC}$ coils, with $N I_{a c 1}=N I_{a c 2}$. The circuit is again completely balanced, with the flux paths between the two AC coils no longer significant. The equivalent circuit for the test is shown in Fig. 5. The nominal direction of each flux path is as indicated in Fig. 5. The flux linkage of each coil is again measured during the test.

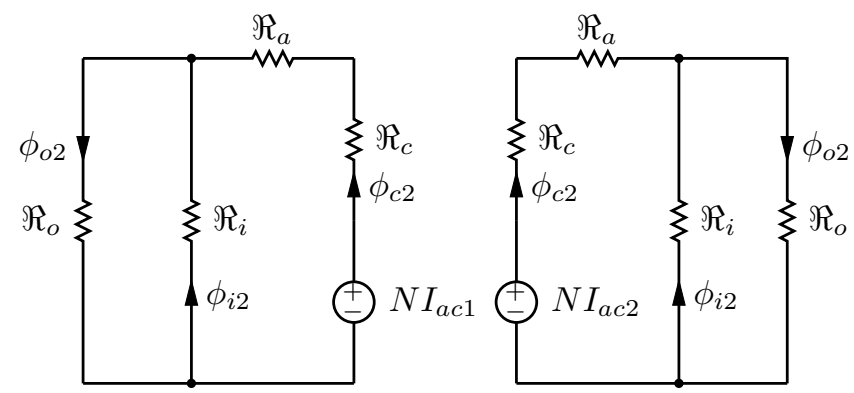

Fig. 5. Equivalent Circuit for Test 2

Through simple circuit analysis:

$$
\begin{aligned}
\Re_{i}\left(\phi_{o 2}-\phi_{c 2}\right) & =-\Re_{o} \phi_{o 2} \\
\text { and } \Re_{c a} & =\frac{\Re_{i}\left(\phi_{o 2}-\phi_{c 2}\right)+N I_{a c 1}}{\phi_{c 2}}
\end{aligned}
$$

Again $\phi_{o 2}$ is equal to one half of the measured flux linkage of the DC coil and $\phi_{c 2}$ is equal to the measured flux linkage of either AC coil.

Solving (1) and (3) simultaneously results in:

$$
\begin{gathered}
\Re_{i}=\frac{N I_{d c}}{\phi_{o 1}-\phi_{c 1}-\frac{\phi_{o 1}}{\phi_{o 2}}\left(\phi_{o 2}-\phi_{c 2}\right)} \\
\Re_{o}=\frac{N I_{d c}}{\phi_{o 1}-\phi_{o 2} \frac{\phi_{o 1}-\phi_{c 1}}{\phi_{o 2}-\phi_{c 2}}}
\end{gathered}
$$

Note that all of the elements in (5) and (6) are either measured flux linkage values or an applied mmf. Hence, $\Re_{i}$ and $\Re_{o}$ can be completely determined from Tests 1 and 2 . The series reluctance $\Re_{c a}$ can be calculated by substituting $\Re_{i}$ from (5) into either (2) or (4).

\section{Test 3}

In Test 3 the sources of mmf are again restricted to the AC coils; however, this time with $N I_{a c 1}=-N I_{a c 2}$. For this case the flux paths between the two AC coils, along with the associated reluctance $\Re_{y}$, are significant. Hence, the flux between the two AC coils must also be measured, along with the flux linkage of each coil. In FEA the flux through $\Re_{y}$ can be determined by integrating the flux density across the entire plane between the two coils. The equivalent circuit for this test is shown in Fig. 6. The nominal direction of each flux path is as indicated in Fig. 6. Note that the directions of the corresponding flux paths (on each side of the circuit) are now opposite to each other. This is important when considering the flux linkage of the DC coil, as the net flux linkage will be zero; however, the flux linking each side of the coil is non-zero, but equal and opposite (i.e. $\left|\phi_{o 3 l}\right|=\left|\phi_{o 3 r}\right|$ and $\left.\phi_{d c}=\phi_{o 3 l}+\phi_{o 3 r}=0\right)$.

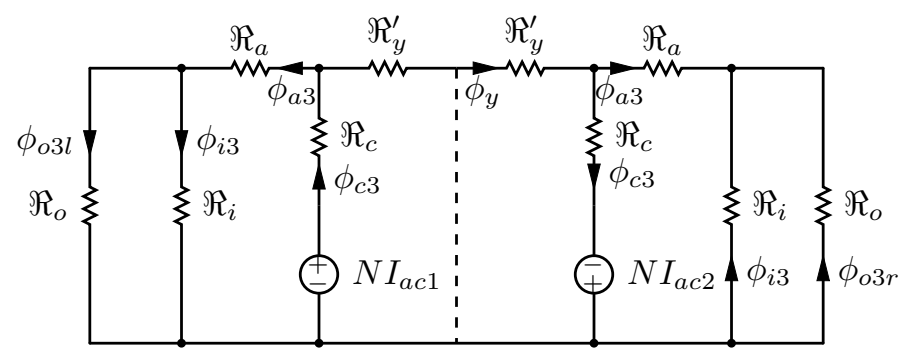

Fig. 6. Equivalent circuit for Test 3

To simplify the circuit of Fig. $6, \Re_{y}$ has been split into two equal reluctances in series (each denoted as $\Re_{y}^{\prime}$ ). Since the circuit is symmetrical, this allows for the two sides of the circuit to be separated - this is possible because the mid point of $\Re_{y}$ is at the same potential as the common node between $N I_{a c 1}$ and $N I_{a c 2}$ (as illustrated by the dashed line in Fig. 6). Hence, the analysis can be confined to a single side of the circuit (as was the case with Tests 1 and 2). A simplified version of the left hand side of Fig. 6 is shown in Fig. 7.

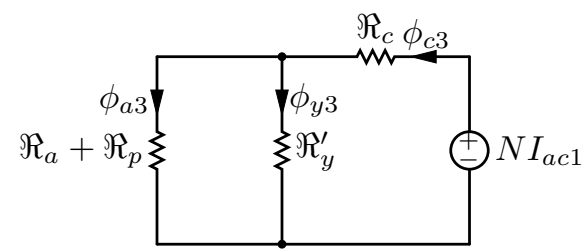

Fig. 7. Simplified Circuit for Test 3

Note that $\Re_{p}$ in Fig. 7 is the parallel combination of $\Re_{i}$ and $\Re_{o}$ (which were both determined via Tests 1 and 2):

$$
\Re_{p}=\frac{\Re_{i} \Re_{o}}{\Re_{i}+\Re_{o}}
$$

Through simple circuit analysis:

$$
\Re_{a}=\frac{N I_{a c 1}-\Re_{c} \phi_{c 3}-\Re_{p}\left(\phi_{c 3}-\phi_{y 3}\right)}{\left(\phi_{c 3}-\phi_{y 3}\right)}
$$


Although all of the flux values in (8) are measured quantities, the equation still contains two unknowns in $\Re_{a}$ and $\Re_{c}$. However, (8) can be re-written in terms of $\Re_{c a}$, which is the series combination of $\Re_{c}+\Re_{a}$ (determined via Tests 1 and 2):

$$
\Re_{a}=\frac{\Re_{c a} \phi_{c 3}+\Re_{p}\left(\phi_{c 3}-\phi_{y 3}\right)-N I_{a c 1}}{\phi_{y 3}}
$$

Hence, the two remaining unknowns $\Re_{c}$ and $\Re_{y}^{\prime}$ can be calculated using (10) and (11) respectively:

$$
\begin{aligned}
\Re_{c} & =\Re_{c a}-\Re_{a} \\
\Re_{y}^{\prime} & =\frac{\left(\phi_{c 3}-\phi_{y 3}\right)}{\phi_{y 3}}\left(\Re_{a}+\Re_{p}\right)
\end{aligned}
$$

\section{Nonlinear Open-Core Model}

The open-core arrangement is a simple extension of the aircore arrangement discussed in Section II, with each of the AC coils now enclosing an iron core (as shown in Fig. 8). This arrangement is considered to be open-core since the return flux paths for both cores are through air. In contrast to the air-core arrangement, this system represents a practical saturated core FCL with the DC coil used to initially drive the cores into saturation.

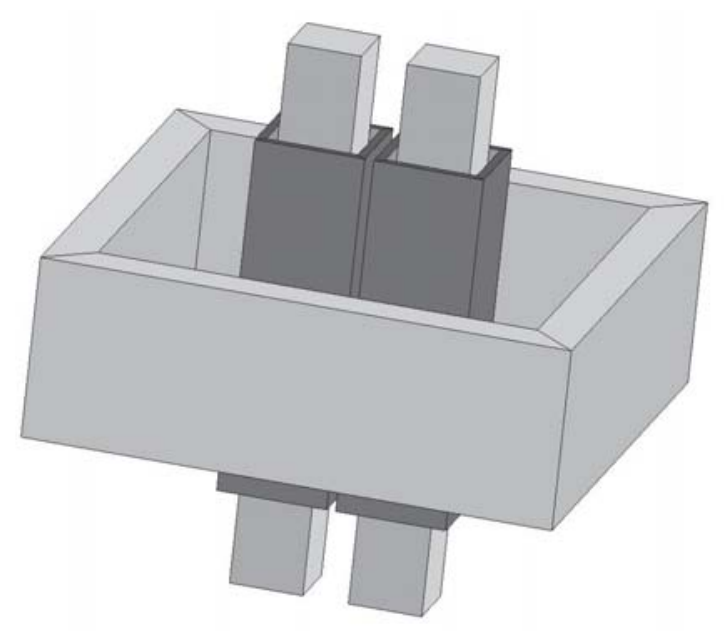

Fig. 8. Single-Phase Open-Core Arrangement

The significant flux paths (and associated reluctances) for the arrangement of Fig. 8 are identified in Fig. 9. As can be seen, these flux paths are identical to those of the air-core arrangement, which were identified in Fig. 2. The principal difference between the two models is that the reluctance of the flux path through the iron cores is nonlinear. Hence, the equivalent magnetic circuit of the open-core arrangement is also identical to that of the air-core arrangement, with the exception being that the core $\left(\Re_{c}\right)$ and yoke $\left(\Re_{y}\right)$ reluctances are nonlinear variables (as shown in Fig. 10). Although the flux path represented by $\Re_{y}$ is theoretically through air, it has been found that this path is influenced by the extremities of the iron core and consequently $\Re_{y}$ has a minor nonlinear component.

The values of the reluctances shown in Fig. 10 can be calculated using the same approach that was used for the

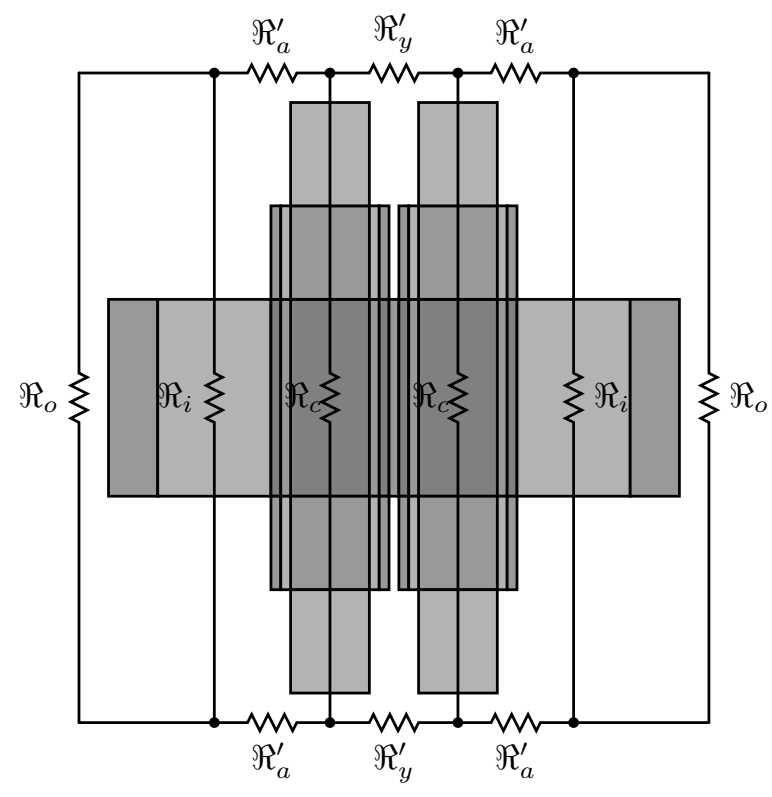

Fig. 9. Equivalent Open-Core Reluctance paths

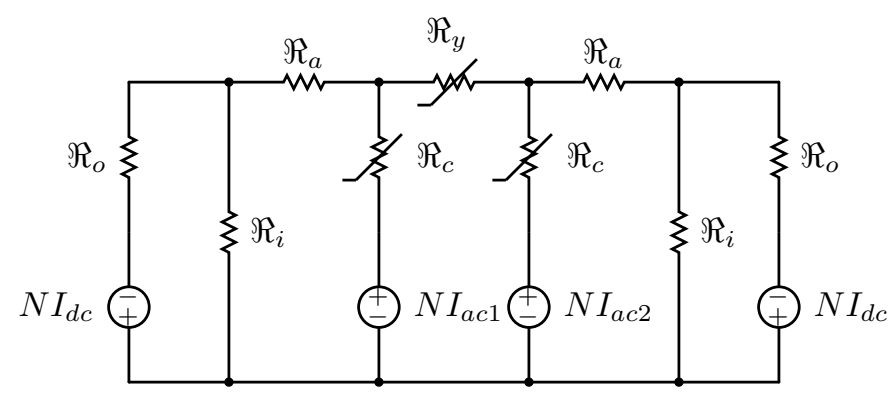

Fig. 10. Equivalent Open-Core Magnetic Circuit

air-core model. In particular, the same three tests are used to determine all of the reluctances. However, there is one significant difference in that the flux measurements (using either FEA or experimentation) need to be undertaken over a range of applied mmf values, since the reluctances are no longer all constant. Hence, the test conditions are:

- Test 1: $N I_{d c}$ is varied, while $N I_{a c 1}=N I_{a c 2}=0$;

- Test 2: $N I_{d c}=0$, while $N I_{a c 1}=N I_{a c 2}$ (varied);

- Test 3: $N I_{d c}=0$, while $N I_{a c 1}=-N I_{a c 2}$ (varied).

During each of these tests the range of applied mmf must be sufficient to saturate the cores.

Test 1 and Test 2 are again used to determine the values of $\Re_{i}$ and $\Re_{o}$. For each of these tests the equivalent circuit is the same as for the corresponding air-core test, with the exception that $\Re_{c}$ is nonlinear. Of particular significance is the fact that the values of $\Re_{i}$ and $\Re_{o}$ are still constants. This means that the method for determining $\Re_{i}$ and $\Re_{o}$ given in Section II (which relied on the principle of superposition) is still valid. Hence, $\Re_{i}$ and $\Re_{o}$ can be determined by (5) and (6) respectively (given previously in Section II). Note also that (2) and (4) from Section II also hold for the series reluctance $\Re_{c a}$; however, the resulting reluctance values are nonlinear since $\Re_{c}$ 
is nonlinear.

Test 3 is used to determine the individual values of $\Re_{c}$ and $\Re_{a}$, along with $\Re_{y}$. The same circuit simplifications that were made for the air-core test are again valid for the open-core case, with the simplified equivalent circuit shown in Fig. 11.

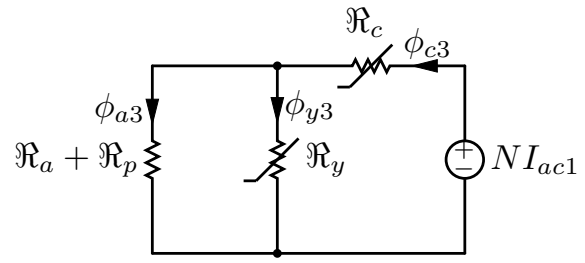

Fig. 11. Simplified Open-Core Circuit for Test 3

Again it can be seen that the simplified equivalent circuit of Fig. 11 is the same as for the corresponding air-core test (Fig. 7), with the exception that $\Re_{c}$ and $\Re_{y}^{\prime}$ are now nonlinear. Although simple circuit analysis leads to the same expression for $\Re_{a}$ as was previously given in (9), the open-core case is complicated by the nonlinear relationship between $\Re_{c a}$ and the mmf acting on it. In particular, since the mmf acting on $\Re_{c a}$ is different in each of Tests 1,2 and 3, care needs to be taken when substituting previously determined values of $\Re_{c a}$ (from Tests 1 or 2) into (9). It is however, still possible to make such substitutions for very low values of applied mmf, since $\Re_{c a}$ is constant below the saturation region. Once $\Re_{a}$ is determined, $\Re_{y}^{\prime}$ can be calculated using (11) and curves for $\Re_{c}$ can be determined for each of Tests 1, 2 and 3 using (10) (see example curves in Fig. 16).

\section{MOdel VAlidation}

\section{A. Air-Core Model}

In order to validate the air-core equivalent model, an example arrangement was simulated using the Magsoft Flux3D FEA package. In this example each of the coils consisted of 120 turns. The AC coils had a cross-sectional area of $0.01 \mathrm{~m}^{2}$ and a length of $390 \mathrm{~mm}$. The DC coils had a cross-sectional area of $0.2304 \mathrm{~m}^{2}$ and a length of $200 \mathrm{~mm}$.

Magnetostatic simulations of Tests 1,2 and 3 were initially undertaken to determine the reluctance values of the equivalent model - the resulting flux linkage values are given in Table I, with the subsequently calculated reluctance values given in Table II.

Table I

Flux LinKage Results From Tests 1, 2 AND 3

\begin{tabular}{cccc}
\hline Value & Test 1 & Test 2 & Test 3 \\
\hline \hline$N I_{d c}$ & $6 \times 10^{4}$ & 0 & 0 \\
$N I_{a c 1}$ & 0 & $6 \times 10^{4}$ & $6 \times 10^{4}$ \\
$N I_{a c 2}$ & 0 & $6 \times 10^{4}$ & $-6 \times 10^{4}$ \\
$\phi_{d c}$ & $4.08 \times 10^{-2}$ & $2.59 \times 10^{-3}$ & 0 \\
$\phi_{a c 1}$ & $1.29 \times 10^{-3}$ & $1.85 \times 10^{-3}$ & $1.99 \times 10^{-3}$ \\
$\phi_{a c 2}$ & $1.29 \times 10^{-3}$ & $1.85 \times 10^{-3}$ & $-1.99 \times 10^{-3}$ \\
$\phi_{y}$ & 0 & 0 & $1.83 \times 10^{-3}$ \\
\hline
\end{tabular}

Table II

Equivalent Model Reluctance Values

\begin{tabular}{cc}
\hline Reluctance & Value \\
\hline \hline$\Re_{i}$ & 2143870 \\
$\Re_{o}$ & 930192 \\
$\Re_{c}$ & 29965839 \\
$\Re_{a}$ & 1732886 \\
$\Re_{y}$ & 407078 \\
\hline
\end{tabular}

After determining the reluctance values, the performance of the analytical air-core equivalent model was then compared with FEA simulations under transient conditions. These tests involved applying different currents to each of the coils and comparing the resulting flux linkage of each coil. The numerical computing environment Matlab was used to calculate the resulting flux linkage values for the analytical model, while a transient FEA solution of the example system was obtained using Magsoft FLUX3D. The test conditions are summarised in Table III, with the resulting flux transients shown in Fig. 12.

Table III

Test CONDitions For Model VALIDATION

\begin{tabular}{cc}
\hline Coil Current & Test Value (A) \\
\hline \hline$I_{d c}$ & 10 \\
$I_{a c 1}$ & $100 \sin (t)$ \\
$I_{a c 2}$ & $50 \sin (2 t)$ \\
\hline
\end{tabular}

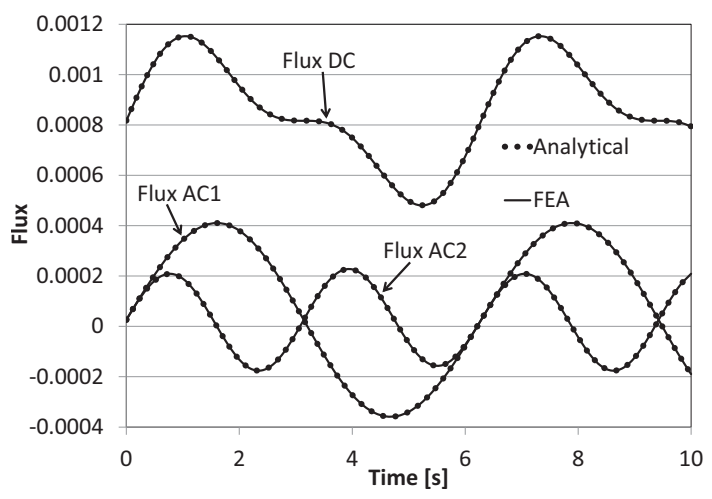

Fig. 12. Flux Comparison for Air-Core Model

The comparisons shown in Fig. 12 demonstrate that the analytical air-core equivalent model produces results that are practically identical to the FEA solution. Different AC coil currents (i.e. $I_{a c 1} \neq I_{a c 2}$ ) were chosen for these tests in order to highlight the $\mathrm{AC}$ to $\mathrm{DC}$ coupling. It is clear from Fig. 12 that this coupling is effectively predicted by the analytical model.

\section{B. Open-Core Model}

In a similar manner to the air-core case, an example opencore arrangement was simulated using the Magsoft Flux3D FEA package. The iron cores in this example had a crosssectional area of $0.0064 \mathrm{~m}^{2}$ and a length of $600 \mathrm{~mm}$. All of the coils were the same as described in Section IV-A. 
Again, magnetostatic simulations of Tests 1, 2 and 3 were initially undertaken to determine the reluctance values of the equivalent model. Unlike the air-core case though, each test required multiple static solutions as the applied mmf was varied. The resulting flux linkage values for Tests 1,2 and 3 are shown in Figures 13, 14 and 15 respectively, with the subsequently calculated reluctance values shown in Fig. 16.

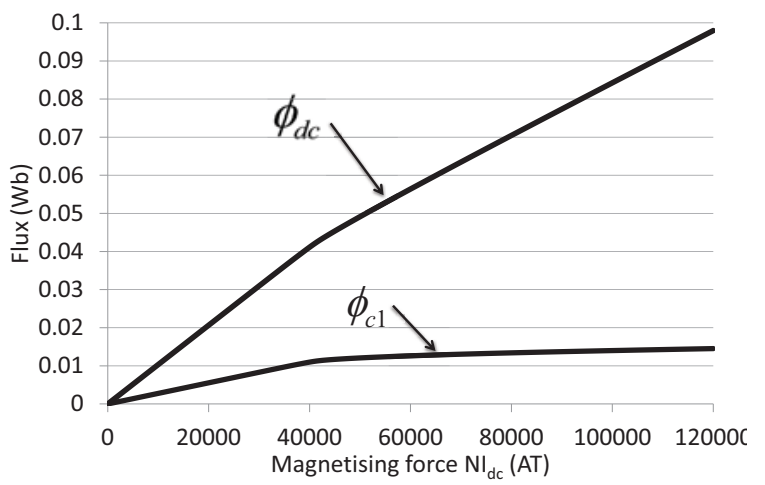

Fig. 13. Flux Linkage - Test 1

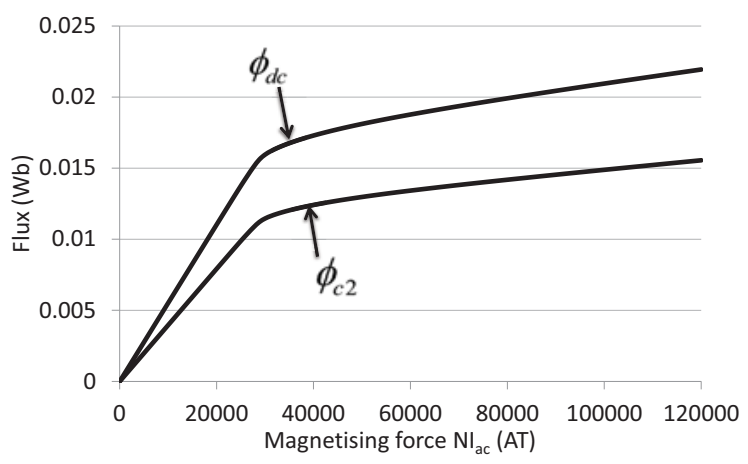

Fig. 14. Flux Linkage - Test 2

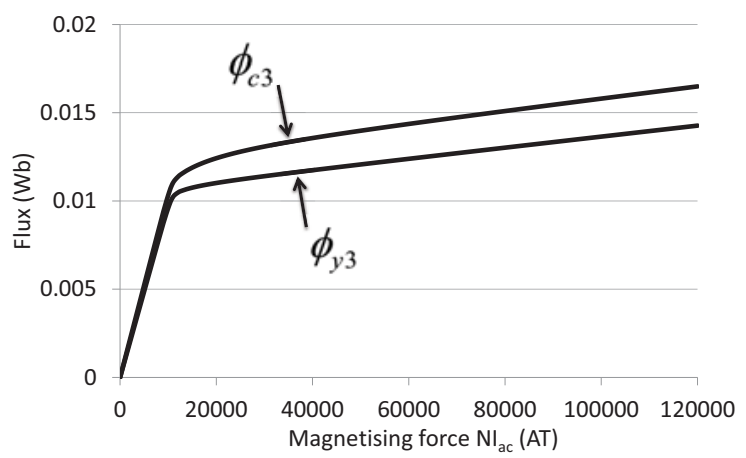

Fig. 15. Flux Linkage - Test 3

In Fig. 16 the calculated reluctance values are plotted against the source mmf of each test. As can be seen, $\Re_{a}, \Re_{i}$ and $\Re_{O}$ are practically constant (as expected). In contrast, $\Re_{c}$ and $\Re_{y}$ are constant at low values of applied mmf and then increase as the iron becomes saturated. The change in $\Re_{y}$ as the iron saturates is quite small (and in some cases can be approximated as a constant reluctance), whereas $\Re_{c}$ increases

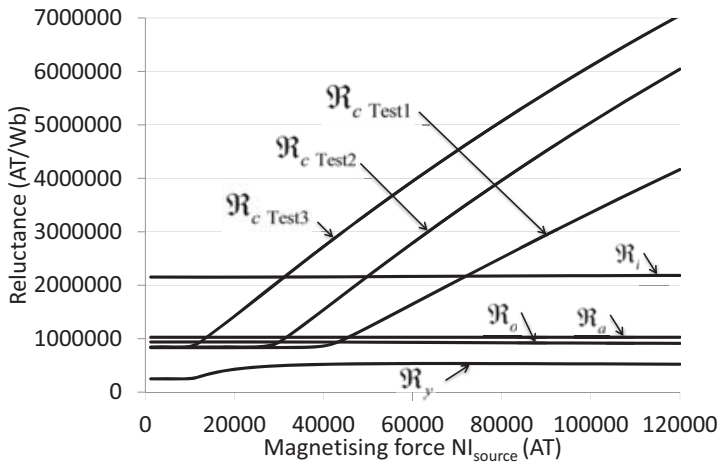

Fig. 16. Reluctance vs Source mmf

rapidly (approaching the equivalent air-core reluctance). Note also that since each reluctance in Fig. 16 is plotted against the source mmf of each test, the plots of $\Re_{c}$ appear to show different saturation points for each of the tests. If $\Re_{c}$ is instead plotted against the component of mmf directly acting on the associated flux path (i.e. through the cores), the resulting values for each test are uniform as shown in Fig. 17.

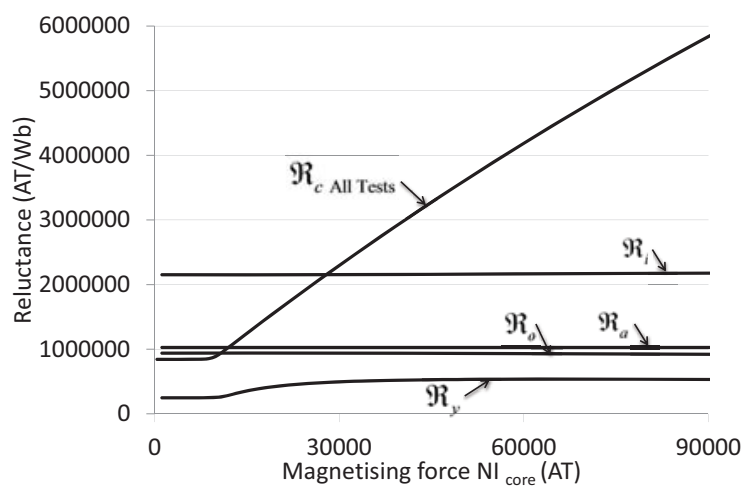

Fig. 17. Reluctance vs mmf of Relevant Flux Path

After determining the reluctance values, the performance of the analytical open-core model was also compared with FEA simulations under transient conditions. In this test the simulated AC coils were wound in opposite directions and connected in series (as is normally the case in a saturated core FCL). Also, the current applied to the DC coil was chosen to be sufficient to saturate the coils, while the current applied to the AC coils was chosen to replicate the core de-magnetisation during a fault event. Matlab was again used to calculate the resulting flux linkage values for the analytical model, while a transient FEA solution of the system was obtained using Magsoft FLUX3D. The actual test conditions are summarised in Table IV.

Table IV

TEST CONDITIONS FOR OPEN-CORE VALIDATION

\begin{tabular}{cc}
\hline Coil Current & Test Value (A) \\
\hline \hline$I_{d c}$ & 330 \\
$I_{a c}$ & $400 \sin (t)$ \\
\hline
\end{tabular}

When using Matlab with the analytical model, the flux link- 
age calculations can be simplified through circuit reductions via Thévenin's theorem. Although the circuit is nonlinear, the Matlab calculations are performed at discrete time instances with single values of $\Re_{c}$ calculated for each instance. Hence, the Thévenin approach remains valid at each time instant. It should also be noted that the mmf acting on the cores $\left(N I_{\text {core }}\right)$ must be known at each time instant, in order to accurately calculate the relevant values of $\Re_{c}$ in each core. However, since $\Re_{c}$ must also be known to determine $N I_{\text {core }}$, the value of $N I_{\text {core }}$ from the previous time instant is used to determine $\Re_{c}$. As long as the chosen time-step is sufficiently small, this approach produces a relatively accurate approximation of circuit flux.

The first circuit reduction can be obtained by considering $N I_{d c}$ as the only source and the entire central core section of Fig. 10 as the load. Hence, via Thévenin's theorem Fig. 10 can be reduced to the circuit shown in Fig. 18.

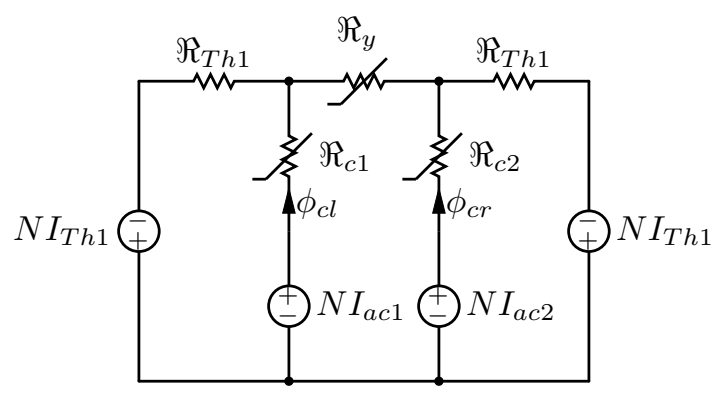

Fig. 18. Initial Open-Core Circuit Reduction

where:

$$
\Re_{T h 1}=\frac{\Re_{a}\left(\Re_{o}+\Re_{i}\right)+\Re_{o} \Re_{i}}{\Re_{o}+\Re_{i}}
$$

and

$$
N I_{T h 1}=\frac{\Re_{i}}{\Re_{i}+\Re_{o}} N I_{d c}
$$

If $\Re_{c 1}$ is then considered as the load, further successive reductions can be made as shown in Fig. 19 and Fig. 20.

In Fig. 19:

$$
\Re_{T h 2}=\frac{\Re_{y}\left(\Re_{T h 1}+\Re_{c 2}\right)+\Re_{T h 1} \Re_{c 2}}{\Re_{T h 1}+\Re_{c 2}}
$$

and

$$
N I_{T h 2}=\frac{\Re_{T h 1} N I_{a c 2}-\Re_{c 2} N I_{T h 1}}{\Re_{T h 1}+\Re_{c 2}}
$$

In Fig. 20:

$$
\Re_{T h 3}=\frac{\Re_{T h 1} \Re_{T h 2}}{\Re_{T h 1}+\Re_{T h 2}}
$$

and

$$
N I_{T h 3}=N I_{a c 1}+\frac{\Re_{T h 2} N I_{T h 1}-\Re_{T h 1} N I_{T h 2}}{\Re_{T h 1}+\Re_{T h 2}}
$$

Hence, the flux linkage of AC coil 1 is:

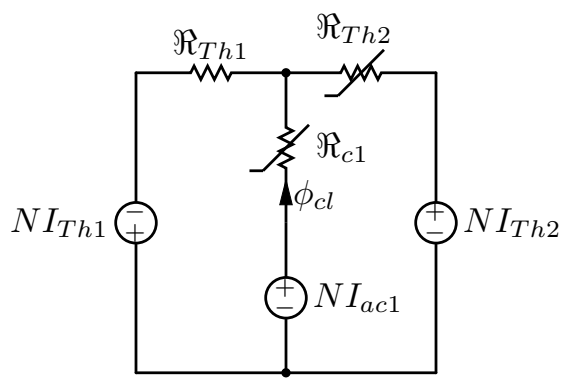

Fig. 19. Open-Core Circuit Reduction with $\Re_{c 1}$ as load

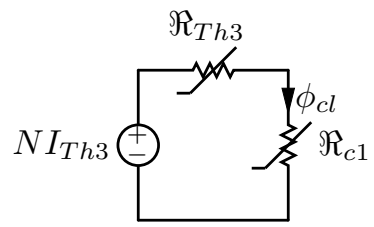

Fig. 20. Final Open-Core Circuit Reduction with $\Re_{c 1}$ as load

$$
\phi_{c l}=\frac{N I_{T h 3}}{\Re_{T h 3}+\Re_{c 1}}
$$

The same approach can be taken when considering $\Re_{c 2}$ as the load, which leads to:

$$
\Re_{T h 4}=\frac{\Re_{y}\left(\Re_{T h 1}+\Re_{c 1}\right)+\Re_{T h 1} \Re_{c 1}}{\Re_{T h 1}+\Re_{c 1}}
$$

$$
\begin{gathered}
N I_{T h 4}=\frac{\Re_{T h 1} N I_{a c 1}-\Re_{c 1} N I_{T h 1}}{\Re_{T h 1}+\Re_{c 1}} \\
\Re_{T h 5}=\frac{\Re_{T h 1} \Re_{T h 4}}{\Re_{T h 1}+\Re_{T h 4}} \\
N I_{T h 5}=N I_{a c 2}-\frac{\Re_{T h 1} N I_{T h 4}}{\Re_{T h 1}+\Re_{T h 2}}+\frac{\Re_{T h 2} N I_{T h 1}}{\Re_{T h 1}+\Re_{T h 2}}
\end{gathered}
$$

And the flux linkage of AC coil 2 is:

$$
\phi_{c r}=\frac{N I_{T h 5}}{\Re_{T h 5}+\Re_{c 2}}
$$

The flux linkage of the DC coil is:

$$
\phi_{d c}=\phi_{o l}+\phi_{o r}
$$

where:

$$
\begin{aligned}
& \phi_{o l}=\frac{\Re_{i}\left(N I_{a c 1}+N I_{T h 1}-\phi_{a c 1} \Re_{c 1}\right)}{\Re_{T h 1}\left(\Re_{i}+\Re_{o}\right)}+\frac{N I_{d c}}{\Re_{i}+\Re_{o}} \\
& \phi_{o r}=\frac{\Re_{i}\left(N I_{a c 2}+N I_{T h 1}-\phi_{a c 2} \Re_{c 2}\right)}{\Re_{T h 1}\left(\Re_{i}+\Re_{o}\right)}+\frac{N I_{d c}}{\Re_{i}+\Re_{o}}
\end{aligned}
$$

The resulting flux transients from both the analytical model and the FEA simulation (for the test conditions summarised in Table IV) are compared in Fig. 21. The results show that the output of the analytical model is practically identical to that 
of the FEA solution. Notice also that during the positive half $\mathrm{AC}$ cycle one core de-saturates while the other core remains in saturation. During the negative half AC cycle, the second core de-saturates as the first remains in saturation. The results also show the analytical model effectively predicted the $\mathrm{AC}$ to DC coupling in the system.

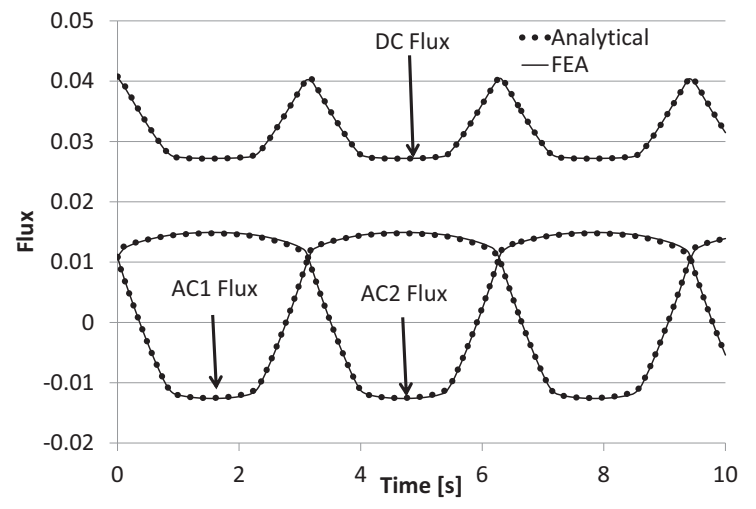

Fig. 21. Comparison of Analytical and FEA

\section{CONCLUSIONS}

A nonlinear reluctance model of a saturated core FCL has been presented in this paper. This model is based on the magnetic circuit concept, with the FCL geometry represented by an equivalent circuit that includes all significant flux paths. This model allows for the nonlinear magnetic behaviour of the FCL to be calculated in an analytical manner, which reduces reliance on the time consuming and complex FEA approach. The model of a linear single-phase air-core arrangement was presented first in Section II, with the approach extended to an open-core FCL arrangement in Section III.

The models for both the air-core arrangement and the opencore FCL arrangement were validated in Section IV. In both cases the analytical models were used to calculate the flux linkage of each coil under transient current conditions. The calculated flux linkage in each case was then compared with FEA simulations under the same conditions. As shown in Sections IV-A and IV-B the outputs of the analytical models were practically identical to each of the corresponding FEA solutions. The results presented in Sections IV-A and IV-B also demonstrated that this modelling approach inherently accounted for the AC to DC coupling effect present in both systems.

One important aspect of the presented modelling approach, is that the basic magnetic circuit is the same for both the aircore arrangement and the open-core FCL arrangement. The only significant difference is that the reluctance of the flux path through the cores is constant in the air-core model and nonlinear in the open-core FCL model. This approach can be further extended to closed-core FCL arrangements. Again, the basic magnetic circuit is the same for a closed-core FCL; however, additional reluctances (primarily $\Re_{a}$ and $\Re_{o}$ ) are nonlinear. These additional non-linearities add complexity to the determination of the reluctance values of the analytical model. Further research is currently underway to evaluate whether the three tests identified in Section II are still sufficient to determine all of the reluctance values in the closed-core case.
The results presented in this paper covered the analytical calculation of magnetic flux in a saturated core FCL. Further development of coupling the magnetics to the electric circuit will allow the reluctance model to be integrated into network simulation packages, to analytically determine the electrical characteristics of saturated core FCLs. Currently, the model is being integrated with the PSCAD simulation package to provide a complete network solution.

\section{REFERENCES}

[1] E. I. . A. O. of Communications, "International energy outlook 2011," tech. rep., U.S. Energy Information Adminstration, EI-40 U.S. Energy Information Adminstration Forrestal Building Washington DC 20585, 2011.

[2] J. Moscrop and F. Darmann, "Design and development of a 3-phase saturated core high temperature superconducting fault current limiter," in Electric Power and Energy Conversion Systems, 2009. EPECS '09. International Conference on, pp. 1 -6, nov. 2009.

[3] F. Darmann and T. Beales, "New fault current limiters for utility substations - design, analysis, construction and testing," in TechCon Asia-Pacific Conference, (Sydney, Australia), 2003.

[4] C. J. Hawley, F. Darmann, and T. P. Beales, "Performance of a $1 \mathrm{mv}$ a high temperature superconductors-enabled saturable magnetic core-type fault current limiter," Superconductor Science and Technology, vol. 18, pp. 255-259, 2005.

[5] Y. He, J. Li, X. Zong, J. Sun, Y. Wang, C. Wu, and J. Wang, "The high voltage problem in the saturated core hts fault current limiter," Physica C: Superconductivity, vol. 386, no. 0, pp. 527 - 530, 2003.

[6] F. Moriconi, N. Koshnick, F. de la Rosa, and A. Singh, "Modeling and test validation of a $15 \mathrm{kv} 24 \mathrm{mva}$ superconducting fault current limiter," in Transmission and Distribution Conference and Exposition, 2010 IEEE PES, pp. 1 - 6, 2010.

[7] S. B. Abbott, D. A. Robinson, S. Perera, F. A. Darmann, C. J. Hawley, and T. P. Beales, "Simulation of hts saturable core-type fcls for mv distribution systems," IEEE Transactions on Power Delivery, vol. 20, no. 2, 2006.

[8] E. R. Laithwaite, Exciting Electrical Machines, pp. 2 - 5. Pergamon Press Ltd., 1974.

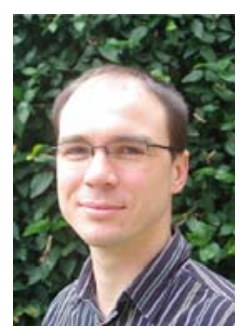

Philip A. Commins received the B.E. (Mechatronics) degree with first class honours from The University of Wollongong, Wollongong, Australia in 2006. In 2007, he commenced his Ph.D. degree at The University of Wollongong in high precision tubular linear motors and is currently finalising his doctoral thesis part-time. In 2011, he joined the Fault Current Limiter research group as a full time research fellow at the University of Wollongong, working on modelling, optimising, designing and experimentally testing saturated core Fault Current Limiters. His work has included extensive magnetic modelling of saturated core FCLs using 3D Finite Element Analysis techniques.

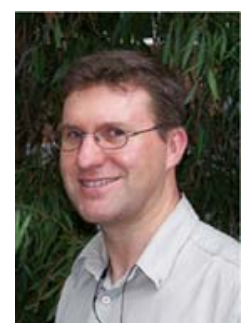

Jeffrey W. Moscrop received the B.E. (Hons.) and Ph.D. degrees from the University of Wollongong in 1998 and 2008 respectively. He is currently a fulltime research fellow and leader of the Fault Current Limiter (FCL) research group at the University of Wollongong. Since the year 2000, he has been involved in the design and development of complex experimental test-beds for research purposes, including linear servo-motor test-beds, magnetically impelled arc butt welding test-beds and high temperature superconducting test-beds. In 2008 he set-up Australia's first dedicated FCL testing facility at the University of Wollongong. To date this facility has tested and characterized over 100 different FCL core and coil designs. He has also been involved in multiple commercial FCL designs at voltage levels ranging from $11 \mathrm{kV}$ to $120 \mathrm{kV}$. 